
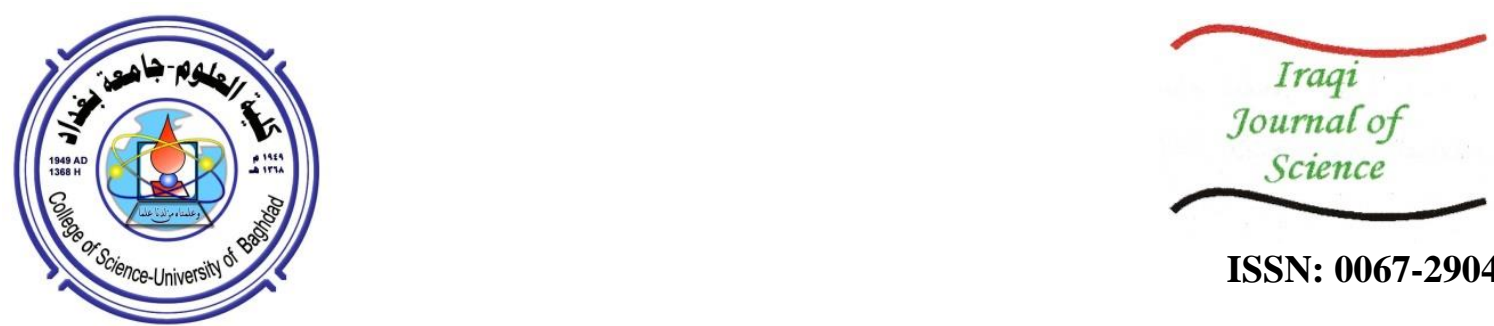

ISSN: 0067-2904

\title{
A Decision Tree-Aware Genetic Algorithm for Botnet Detection
}

\author{
Thurayaa B. Alhijaj*, Sarab M. Hameed, Bara'a A. Attea \\ Department of Computer Since, Collage of Since, University of Baghdad, Baghdad, Iraq
}

Received: 25/7/2020 Accepted: 14/10/2020

\begin{abstract}
In this paper, the botnet detection problem is defined as a feature selection problem and the genetic algorithm (GA) is used to search for the best significant combination of features from the entire search space of set of features. Furthermore, the Decision Tree (DT) classifier is used as an objective function to direct the ability of the proposed GA to locate the combination of features that can correctly classify the activities into normal traffics and botnet attacks. Two datasets namely the UNSW-NB15 and the Canadian Institute for Cybersecurity Intrusion Detection System 2017 (CICIDS2017), are used as evaluation datasets. The results reveal that the proposed DT-aware GA can effectively find the relevant features from the whole features set. Thus, it obtains efficient botnet detection results in terms of F-score, precision, detection rate, and number of relevant features, when compared with DT alone.
\end{abstract}

Keywords: Botnet; decision tree; feature selection; genetic algorithm.

$$
\text { شجرة القرار مع خوارزمية جينية لاكتشاف شبكة الروبوت }
$$

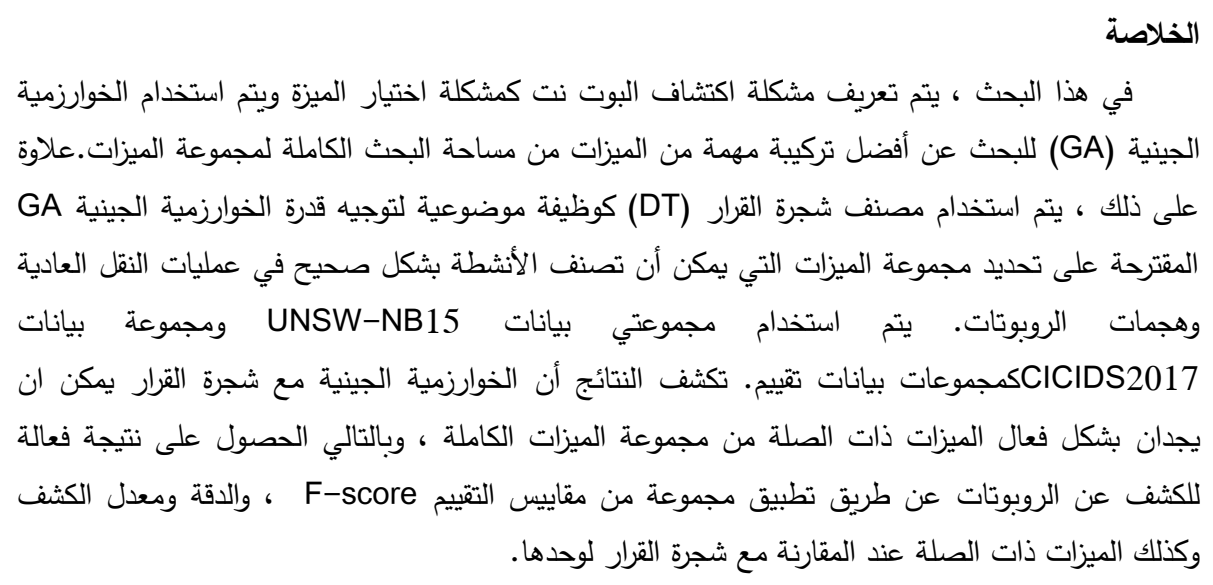

\section{Introduction}

Botnets, as a set of the words "ro-bot" and "net-work", is a network of computers infected with malicious software and remotely commanded and controlled by cybercriminals, or the so-called 
botmasters. Any host on the Internet that is flawed by a botmaster becomes a zombie in this botnet and joins tens or even hundreds of thousands of zombies in the botnet's army.

The main Command and Control $(\mathrm{C} \& \mathrm{C})$ servers of the intruders in this army will, in turn, gain huge amount of money while accessing large numbers of infected machines. Due to the vast usage of network-based services and applications in our daily digital world, such cybercriminal activities significantly affect the economic cost. In April 2012, e.g., the Symantec cybercrime report reported that cyber-attacks cost US $\$ 114$ billion each year. However, if the time lost by companies trying to recover from cyber-attacks is also counted, the total cost of cyber-attacks would reach US $\$ 385$ billion [1]

A Botnet can emerge from several families; namely Internet Relay Chat (IRC), Hypertext Transfer Protocol (HTTP), and Peer-to-Peer (P2P). Based on the C\&C infrastructure, a botnets network can be classified into centralized and decentralized. In centralized botnets, the botmaster usually uses the $\mathrm{C} \& \mathrm{C}$ server to send a command to the bots. The centralized botnet is popularly adopted by various botnet families because of its simplicity. However, its main limitation is its single point of the failure $\mathrm{C} \& \mathrm{C}$ server. To evade this defect, decentralized $\mathrm{C} \& \mathrm{C}$ infrastructures, such as the $\mathrm{P} 2 \mathrm{P}$, have been developed by botnet attackers [2].

Although various machine learning and data mining algorithms have been proposed in the literature to build several botnet detection models, almost all these models and algorithms are based on feature extraction (or feature construction), where different feature sets are extracted from the available high dimensional dataset based on some expertise and skills [3]. On the other hand, feature selection, which engages an essential role in developing different machine learning models, is found to be of little attention in the literature of botnet detection.

The contribution of this paper is to present the botnet detection problem as an optimization problem and to adopt a Genetic Algorithm (GA) to tackle it. The problem is formulated as feature selection, where the GA has to search, from a set of different scenarios, for the best possible combination of features that can correctly discriminate botnet traffic data from normal ones. The proposed GA is designed to be within DT-aware search algorithm, where the DT classifier is used to rank the individual solutions according to the F-score rate of botnets.

The remainder of this paper is organized as follows: Section 2 presents some botnet detection related works. Section 3 presents the preliminary concepts. The proposed DT-aware GA for botnet detection is introduced in Section 4. Experimental results and the related discussions are reported in Section 5. Finally, conclusions and possible future ideas are commented in Section 6.

\section{Related work}

Recently, many works have been presented in the literature to describe different botnet detection techniques for botnet detection problems. An anomaly detection model based on the genetic neural feed-forward network is proposed in [4]. Searching for the best setting for the initial weights of backpropagation feed-forward neural networks is the main concept of this model. The work in [5] introduced a botnets detection method to distinguish between two behaviors: bots having non-periodic and normal traffics which normally reveal periodic behavior. Principal components analysis (PCA) is used for feature selection and $\mathrm{J} 48$ algorithm is used for classification. Feature selection from PCA helps in increasing the detection rate of the bot traffic.

In [6] the UNSW-NB15 dataset is analyzed statistically and practically. Five different classifiers: Naïve Bayes (NB), Decision Tree (DT), Artificial Neural Network (ANN), Logistic Regression (LR), and Expectation-Maximization (EM) Clustering are used to assess the complexity in terms of accuracy and False Alarm Rrate (FAR).

To investigate the impact of feature selection on the performance of a botnet detection technique, the study in [2] is suggested. Three different feature selection methods have been developed in this study, namely linear models penalized with the L1 norm, Recursive Feature Elimination (RFE), and Treebased feature selection (i.e. random forest feature ranking). Four main steps constituted the detection methodology. Feature extraction using Tranalyzer is applied to the raw network captures. The statistical feature set being extracted from the first step is then delivered to the selection process to select the most deputy features subset influencing accurate classification of traffic category into botnet and normal ones. The adopted classifiers are used in the third step, while the evaluation of the classification methods is computed in the final step.

In [7], a single objective GA is used to explore the high space of candidate features and attempt to 
locate the best subset that could improve the performance of the GA-based botnet detection system. In [8], a botnet flow classification system based on C4.5 algorithm is described. A set of features is deterministically selected from the input Packet Capture (PCAP) file using the greedy search mechanism of Consistency Subset Evaluation. To extract the behavior pattern of each flow traffic, the classifier algorithm classifies similar flow traffic into groups. The proposed system not only can analyze P2P botnets, but further it extracts the patterns to an application layer and can analyze botnets using HTTP protocols.

A P2P botnet detection method based on four-layer network traffic classification is proposed in [9]. Feature reduction is gained using a decision tree algorithm. While the first layer filters out non P2P packets, the second layer characterizes the captured network traffic into non $\mathrm{P} 2 \mathrm{P}$ and $\mathrm{P} 2 \mathrm{P}$. At the third layer, the selected features are further reduced and at the final layer, P2P botnets are detected using decision tree classifier. Little attention has been paid to the role of feature selection in perceiving only the feature set that could get a lower detection rate than other features.

\section{3 .Preliminary concepts}

\subsection{Genetic algorithm}

Genetic algorithm (GA) is a class of adaptive search algorithms in which the main idea is based on natural genetics. GA starts by initializing a population of several individuals. Then, the individuals are evaluated according to the objective function for the given problem. After that, the selection, crossover, and mutation operators are applied to the entire population for generating new, and hopefully better, individuals. Different canonical selection, crossover, and mutation operators are provided in the literature. Examples are binary tournament selection, roulette wheel selection, $n$-point crossover, inversion mutation, and substitution mutation operators. The process of generating new individuals by selection, crossover, and mutation operators continues until a termination condition is justified $[10,11]$.

\subsection{Decision tree}

Decision tree (DT) is a popular method in machine learning. The decision tree models are determined to be the most valuable in the domain of data mining, considering reasonable accuracy with relatively inexpensive computation. The decision tree is defined as a supervised learning that classifies the labeled trained data into a tree or rules. It divides a data domain (node) recursively into two subdomains such that the subdomains have a higher information gain than the node that it was split from. Finding the best split that gives the maximum information gain is the goal of the optimization algorithm in the decision tree-based supervised learning $[12,13]$.

\section{Proposed decision tree-aware genetic algorithm for botnet detection}

In an abstract language, the proposed GA is designed to be as a feature selector algorithm. It should select the most suitable combination of features from a mixed dataset of both normal and botnet traffics. Further, the DT is used as a supervised learning algorithm to direct the GA for the promising set of features in the search space. Feature selection, however, is proved to be a non-deterministic polynomial time hard (NP-hard) problem. The complexity of the problem increases exponentially with the problem dimension.

In this section, the characteristic components of the proposed GA are presented, while tackling the botnet detection problem. The problem is formulated as a feature selection problem. Furthermore, DT is used to judge the ability of the proposed GA to locate the combination of features that can correctly classify the activities into normal and botnet activities. Figure- 1 depicts the general layout of the proposed botnet detection. 


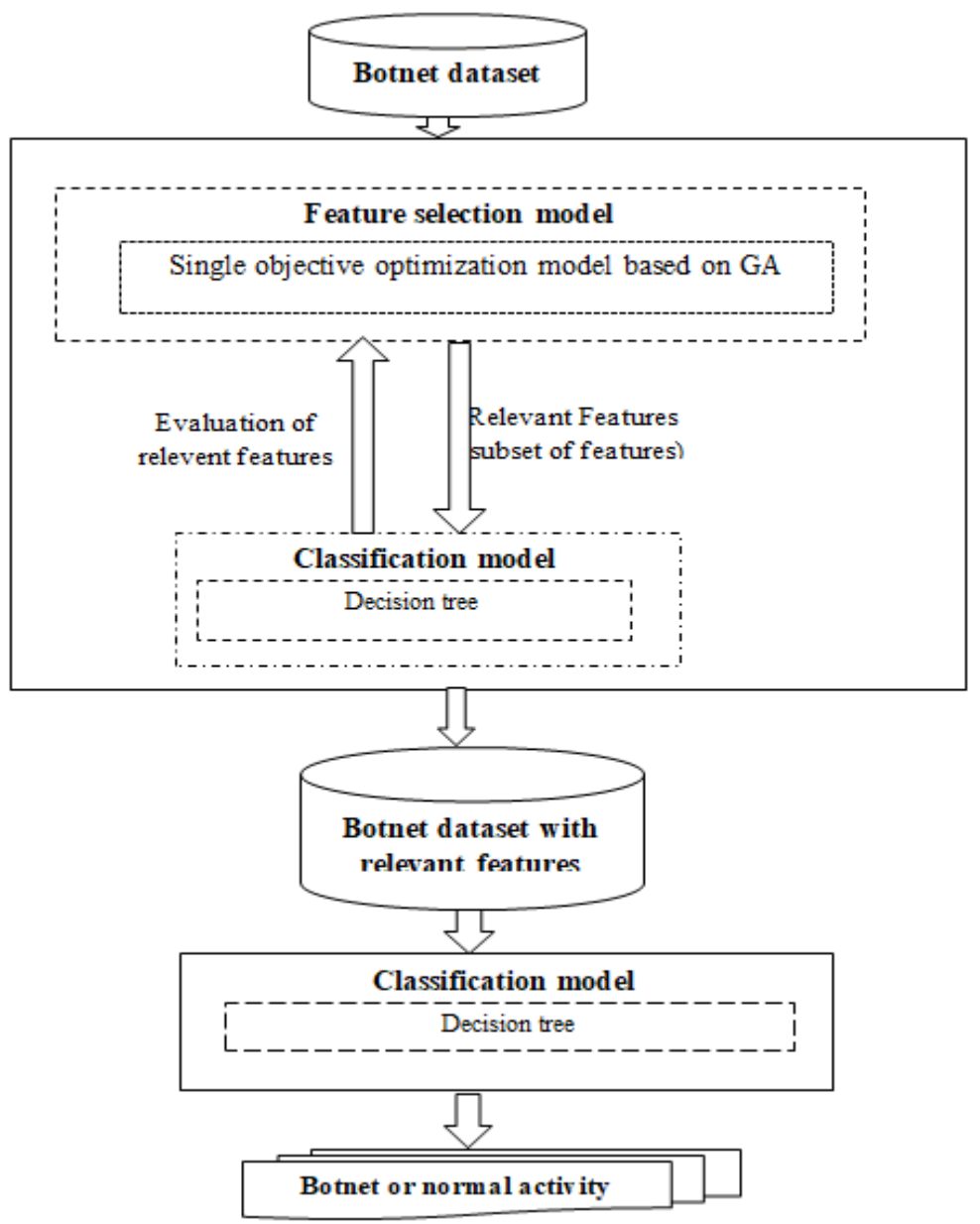

Figure 1- General layout of the proposed botnet detection

\subsection{Individual's genotype encoding and population initialization}

Each individual (solution) in the proposed GA is represented as a fixed length vector of $n$ genes, where $n$ is the total number of features. The locus of each gene maps to the corresponding feature while its allele value can be either 0 or 1 to indicate the absence or presence of this feature. The total search space size for the modeled botnet detection problem can, thus, be calculated as the Cartesian product of presence and absence of all features (i.e., $2^{n}$ ).

GA is a population based algorithm, i.e. it is composed of a population $\mathbb{P}$ of $N$ individuals. The mathematical formulation of $\mathbb{P}$ can be expressed as $\mathbb{P}=\left\{I_{1}, I_{2}, \ldots I_{N}\right\}$. The formulation of the individual $I$ can then be expressed as $I_{1 \leq i \leq N}=\left\{I_{i, 1}, I_{i, 2}, \ldots, I_{i, n}\right\}$. The genes of each individual $I_{i, 1 \leq j \leq n}$ are first generated randomly to ensure unbiased search in the beginning. As expressed in Equation 1, $r$ refers to a uniform random number in the range $[0,1]$ generated for each gene in each individual.

$\forall 1 \leq i \leq N$

$$
I_{i, 1 \leq j \leq n}=\left\{\begin{array}{l}
1 \text { if } r \leq 0.5 \\
0 \text { otherwise }
\end{array}\right.
$$

\subsection{Objective function}

In GA, the objective function $\Phi: I \rightarrow \mathbb{R}$ is used to determine the quality of the individuals during the evolution process. The objective function regarding the feature selection problem within botnet detection $\left(\Phi: \mathrm{I} \rightarrow \mathbb{R}^{+}\right)$is designed to maximize the F-score. F-score is expressed in Eq.2, reflecting the harmonious mean within DR and P. Each individual $I_{i}$ in $\mathbb{P}$ is evaluated according to Equation 2 . The details of evaluating the objective function are sketched in Algorithm 1. 


$$
\begin{aligned}
& \forall i=1, \ldots, N \\
& \quad \Phi\left(I_{i}\right)=\frac{2 * \text { Precision } \times \text { DetectionRate }}{\text { Precision }+ \text { Detection Rate }}
\end{aligned}
$$

Precision (P) as expressed in Eq. 4 measures the truly positive exemplars predicted from the whole number of examples in the positive class.

Detection rate (DR) as expressed in Eq. 3 measures the ratio of positive exemplars that are accurately categorized,

where

$$
\begin{aligned}
& \text { Precision }(P)=\frac{T P}{T P+F P} \\
& \text { Detection Rate }(D R)=\frac{T P}{T P+F N}
\end{aligned}
$$

True positive (TP) represents botnet that is correctly classified,

True negative (TN) represents normal that is correctly classified,

False positive (FP) represents normal that is misclassified as an botnet, and

False negative $(F N)$ represents botnet that is misclassified as normal.

Algorithm 1: Objective Function Evaluation

\section{Input:}

- I: Individual

- $\quad \mathbb{T}$ : training dataset

- $\mathbb{V}$ : validation set

- $\quad L$ : label set of $\mathbb{V}$

- $\quad D$ : No. of traffic data in $\mathbb{V}$

- $\quad n$ : individual length

Output: $\Phi(I)$ : Objective Function value for $I$

1: $\quad$ Parameters Setting

FilteredTrainingSet=[];

FilteredValidationSet $=[]$;

OnFeaturesCounter $=0$;

$T P=0$;

$F P=0$;

$F N=0$;

2: $\quad$ For $i=1$ to $n$

If $I(i)=1$

OnFeaturesCounter $=$ OnFeaturesCounter +1 ;

// Generate Filtered Training set and Filtered Validation set

FilteredTrainingSet $=$ FilteredTrainingSet $\cup \mathbb{T}($ OnFeaturesCounter $)$

FilteredValidationSet $=$ FilteredValidationSet $\cup \mathbb{V}($ OnFeaturesCounter $)$

\section{Endif}

Endfor

3: Apply decision tree classifier for FilteredTrainingSet and assign label for each data in FilteredValidationSet stored in ValidationLabel

4: $\quad$ Compute $T P, F P$ and $F N$

For $j=1$ to $D$

if ValidationLabel $_{j}=1$ and $\boldsymbol{L}_{j}=1$

$T N=T N+1$

else

if ValidationLabel $_{j}=0$ and $\boldsymbol{L}_{j}=1$

$F P=F P+1$

else

$$
\begin{aligned}
\text { if } \text { ValidationLabel }_{j}=1 \text { and } \boldsymbol{L}_{j} & =0 \\
& F N=F N+1
\end{aligned}
$$




\section{Endif \\ Endfor \\ 5: $\quad$ Evaluate individual $I$ using Equation 2}

\subsection{GA evolution operators}

The proposed GA can be described as an iterative transformation function $\Psi: \mathbb{P} \rightarrow \mathbb{P}^{\prime}$ with $\Psi\left(\mathbb{P}_{t}\right)=\mathbb{P}_{t+1}$, where $\mathbb{P}_{t}$ and $\mathbb{P}_{t+1}$ are the population at iteration $t$ and $t+1$, respectively. The population begins within an initial set of individuals and continues till a maximum number of iterations $\max _{g e n}$ have been reached. The evolution (transformation) function $\Psi$ is composed of three operators: selection, crossover, and mutation. Binary tournament selection is adopted in this paper, in which two random individuals are selected and the one with the largest fitness function (largest Fscore) is passed to the mating pool for the next generation.

Uniform crossover operator with probability $p_{c} \in[0,1]$ and mutation operator are used. In uniform crossover, two parents, $I^{p 1}$ and $I^{p 2}$, are crossed over to produce a child, ', as formulated in Equation $(5)$.

$$
\begin{array}{r}
\forall i, 1 \leq i \leq n \\
I_{i}^{\prime}=\left\{\begin{array}{l}
I_{i}^{p 1} \text { if } r \leq 0.5 \\
I_{i}^{p 2} \text { otherwise }
\end{array}\right.
\end{array}
$$

where

$r$ is a uniform random number in $[0,1]$.

For the mutation operator, a random number $p_{m} \in[0,1]$ is generated. The allele of the mutated gene can be flipped from 1 to 0 or vice versa with probability $p_{m}$.

\subsection{Botnet detection based on DT aware GA}

Figure- 1 depicts the general layout incorporating the components of GA mentioned previously. The botnet detection using GA-based feature selection can be recapitulated in Algorithm 2.

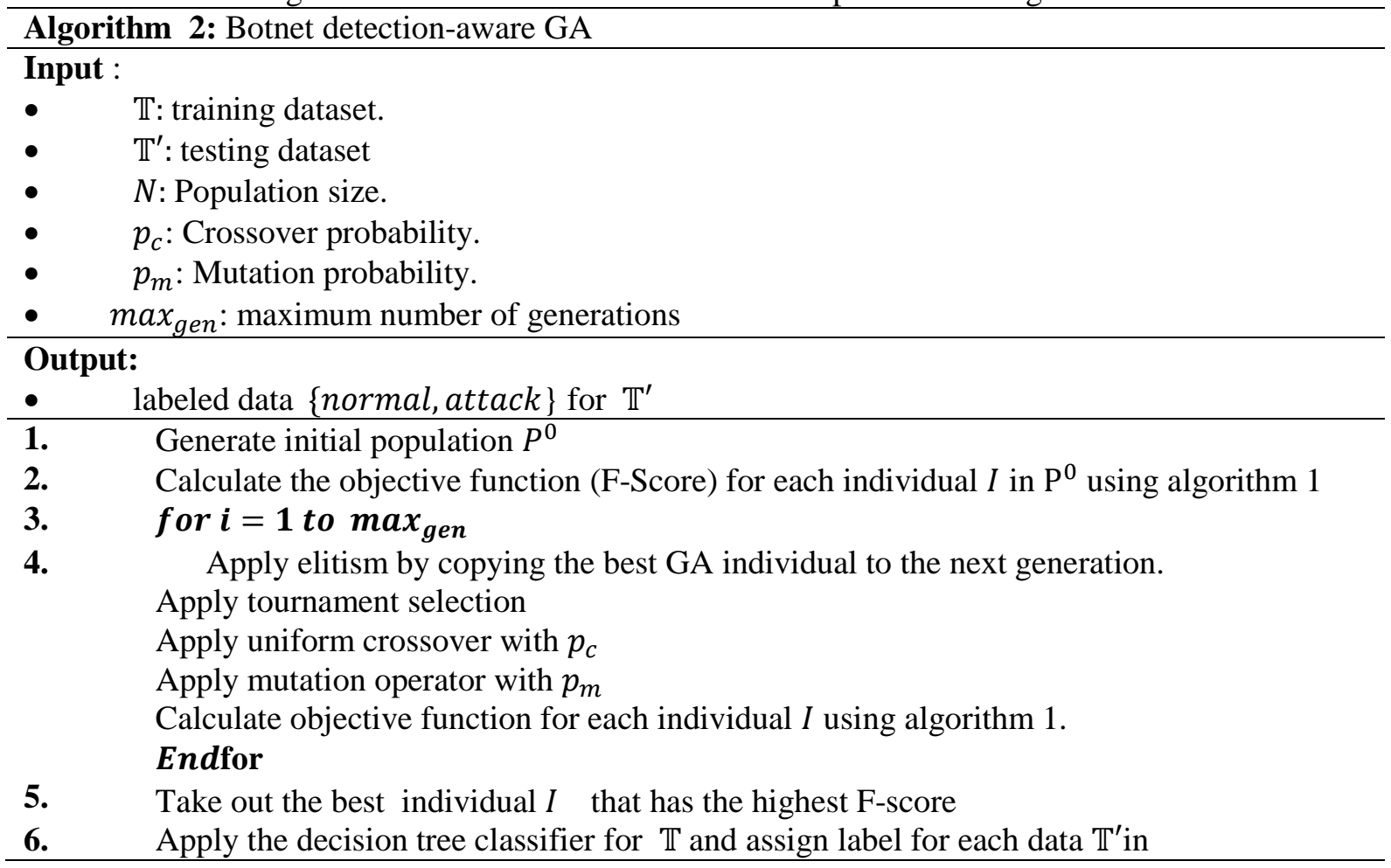




\section{Performance Evaluation}

UNSW-NB15 dataset [6] is used as an evaluation dataset for the assessment of the proposed GAbased botnet detection. The dataset is designed by XIA PerfectStorm tool in the Cyber Range Lab of the Australian Centre for Cyber Security (ACCS) to generate real modern normal and nine types of attack activities. The types are namely Analysis, Backdoor, Denial of service (DoS), Exploits, Fuzzers, Generic, Reconnaissance, Shellcode, and Worms. The dataset is divided into two groups: the first group is the training set containing 175,341 activities and the second is the testing group containing 82,332 activities. The percentage of normal in the training and testing sets is $44.94 \%$ and $31.94 \%$, respectively. While the percentage of the attack in the training and testing sets is $55.06 \%$ and $68.06 \%$, respectively. Each instance in the dataset has 42 features in addition to class labels ( 0 for normal and 1 for attack). Figure- 2 depicts the distribution of the normal and the attack categories in the training and testing sets in UNSW-NB15 dataset.

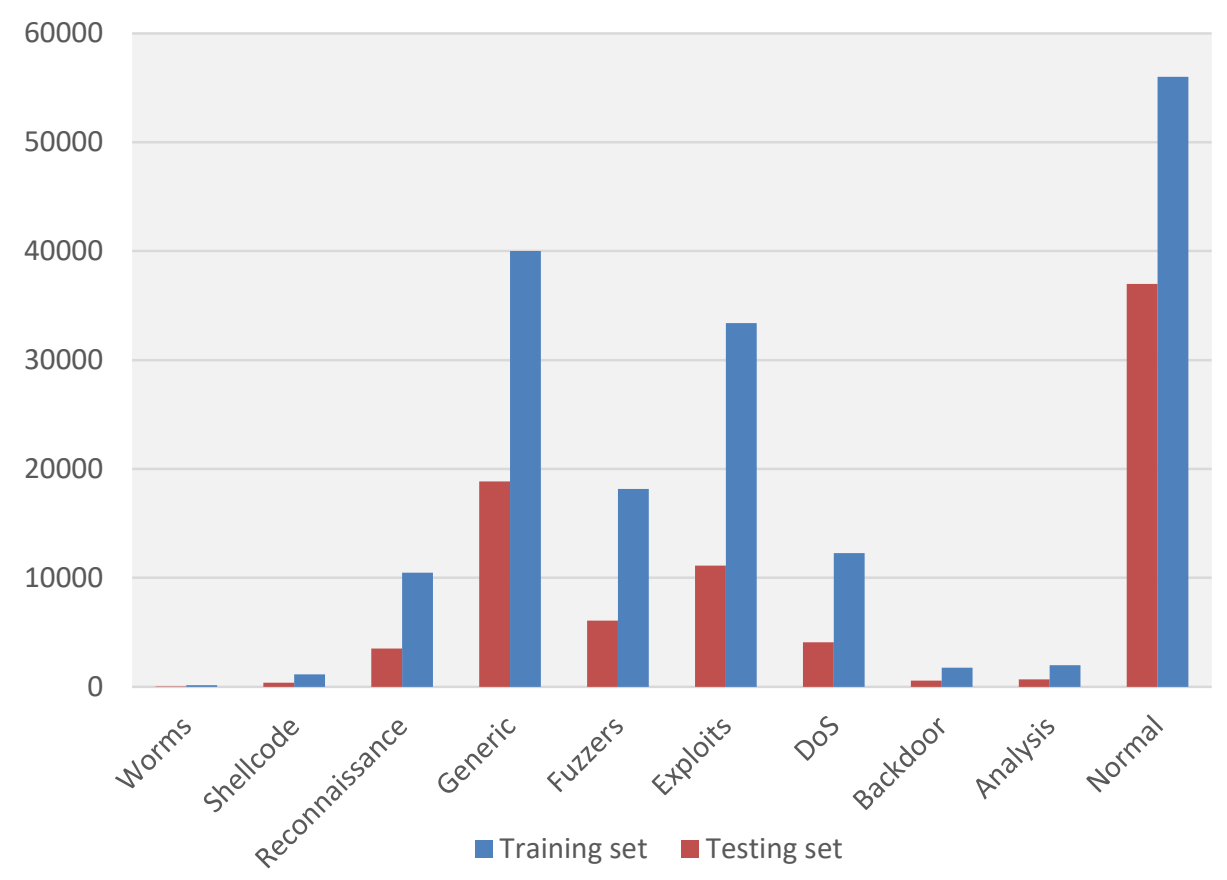

Figure 2- The distribution of the normal and the attack categories in the training and testing sets in UNSW-NB15 dataset.

Furthermore, the CICIDS2017 dataset [14] is used for evaluating the proposed GA botnet detection. The training dataset contains three types of the botnet and the testing dataset includes three types of botnets. Each instance in the dataset has 78 features in addition to class labels (0s for normal traffics and 1s for attacks). The percentage of malicious in the training dataset is $38.33 \%$ while the percentage of malicious flow in the testing dataset is $29 \%$.

Several experiments have been conducted to assess the performance of the proposed GA botnet detection regarding five percentages of validation set $(5 \%, 10 \%, 15 \%, 20 \%$ and $25 \%)$. All the experiments have been conducted with the same setting of GA parameters. The parameters are set as follows: a population of $N=100$ individuals was used and evolved over a sequence of max $_{g e n}=$ $100, p_{c}=0.9$, and $p_{m}=0.1$ have been chosen.

Table-1 reports the $F-$ score, $P$, and $D R$ values of the proposed model, which are evaluated using UNSW-NB15 dataset and represented by an average of 30 runs. Table- 2 reports the average $F-$ score, $P$, and $D R$ values of the proposed model, that are evaluated using CICIDS2017 dataset and represented by an average of 5 runs with the same parameters.

The results in the tables reveal that the proposed GA can effectively find the relevant features from the whole features set, and thus obtaining an efficient botnet detection result. Furthermore, the results highlight that increasing the size of the validation set has a positive impact on achieving high F-score with less number and relevant features. 
Table 1- Comparison of the results of DT against DT-aware GA within UNSW-NB15 dataset

\begin{tabular}{|c|c|c|c|c|}
\hline Model & Avg. FS & Avg. DR & Avg. P & Avg. \# Features \\
\hline DT-42 & 0.9214 & 0.8883 & $\mathbf{0 . 9 5 7 2}$ & 42 \\
\hline DT-GA-\%5 & 0.9374 & 0.9921 & 0.8883 & 14.5667 \\
\hline DT-GA-\% 10 & 0.9368 & 0.9935 & 0.8863 & $\mathbf{1 4 . 4 4 4}$ \\
\hline DT-GA-\%15 & 0.9363 & 0.9928 & 0.8859 & 14.5000 \\
\hline DT-GA-\% 20 & 0.9373 & 0.9933 & 0.8873 & 15.6333 \\
\hline DT-GA-\%25 & $\mathbf{0 . 9 3 8 3}$ & $\mathbf{0 . 9 9 4 2}$ & 0.8884 & 16.966 \\
\hline
\end{tabular}

Table 2- Comparison of the results of DT against DT aware GA within CICIDS2017 dataset

\begin{tabular}{|c|c|c|c|c|}
\hline Model & Avg. FS & Avg. DR & Avg. P & Avg. \# Features \\
\hline DT-78 & 0.9257 & 0.9645 & 0.8900 & 78 \\
\hline DT-GA-\%5 & 0.9715 & 0.9516 & 0.9921 & 36 \\
\hline DT-GA-\%10 & 0.9732 & 0.9516 & 0.9959 & 34 \\
\hline DT-GA-\%15 & 0.9751 & 0.9515 & $\mathbf{0 . 9 9 9}$ & 37 \\
\hline DT-GA-\%20 & 0.9716 & 0.9516 & 0.9923 & 40 \\
\hline DT-GA-\%25 & $\mathbf{0 . 9 8 0 0}$ & $\mathbf{0 . 9 6 8 5}$ & 0.9917 & $\mathbf{3 3}$ \\
\hline
\end{tabular}

\section{Conclusions}

In this paper, a botnet detection model is proposed. The proposed model uses a DT-aware GA to select the best possible subset of features to discriminate botnet traffics from normal traffics. The results reveal the ability of the proposed model to improve the F-score and the detection rate of botnet detection, with less number of features when compared against a DT based botnet detection model. In the future, a multi-objective evolutionary algorithm, such as the decomposition based multi-objective evolutionary algorithm (MOEA/D), can be used to maximize both precision and detection rate, instead of applying a single objective GA.

\section{References}

1. Internet Security Threats Report. Symantec, http://www.symantec.com/threatreport/, last accessed: June 2013.

2. Alauthaman, M., Aslam, N., Zhang, L., Alasem, R., \& Hossain, M. A. 2016. A P2P Botnet detection scheme based on decision tree and adaptive multilayer neural networks. Neural Computing and Applications, 29(11): 991-1004. doi: 10.1007/s00521-016-2564-A.

3. Pektaş, T. Acarman. 2017. Effective Feature Selection For Botnet Detection Based On Network Flow Analysis, International Conference Automatics and Informatics'2017.

4. Yin, C., Awlla, A. H., Yin, Z., \& Wang, J. 2015. Botnet detection based on genetic neural network. International Journal of Security and Its Applications, 9(11): 97-104.

5. Harsha, T., Asha, S., and Soniya, B. 2016. Feature selection for effective botnet detection based on periodicity of traffic. Information Systems Security Lecture Notes in Computer Science, pp. 471-478.

6. Moustafa, N., \& Slay, J. 2016. The evaluation of Network Anomaly Detection Systems: Statistical analysis of the UNSW-NB15 data set and the comparison with the KDD99 data set. Information Security Journal: A Global Perspective, 25(1-3): 18-31. doi: 10.1080/19393555. 2015.1125974.

7. Alejandre, F. V., Cortés, N. C., \& Anaya, E. A. 2017. Feature selection to detect botnets using machine learning algorithms. International Conference on Electronics, Communications and Computers. https://doi.org/10.1109/CONIELECOMP.2017.7891834.

8. Hung, C., \& Sun, H. 2018. A Botnet Detection System Based on Machine-Learning using FlowBased Features. The twelfth international conference on emerging security information, systems 
and technologies,pp. 122-127.

9. Khan, R. U., Zhang, X., Kumar, R., Sharif, A., Golilarz, N. A., \& Alazab, M. 2019. An Adaptive Multi-Layer Botnet Detection Technique Using Machine Learning Classifiers. Applied Sciences, 9(11): 2375. doi: 10.3390/app9112375

10. Aissa, N. B., \& Guerroumi, M. 2015. A genetic clustering technique for Anomaly-based Intrusion Detection Systems. 16th International Conference on Software Engineering, Artificial Intelligence, Networking and Parallel/Distributed Computing. https://doi.org/10.1109 /SNPD.2015.7176182.

11. Dhuha I. M. and Sarab M. H. 2016. A Feature Selection Model based on Genetic Algorithm for Intrusion Detection, Iraqi Journal of Science, Special Issue, Part A, pp. 168-175

12. Suthaharan, S. 2016. Machine Learning Models and Algorithms for Big Data Classification. In Integrated Series in Information Systems, 36. https://doi.org/10.1007/978-1-4899-7641-3

13. Lavanya, D. 2012. Ensemble Decision Tree Classifier For Breast Cancer Data. International Journal of Information Technology Convergence and Services. 2(1): 17-24. https://doi.org/ 10.5121/ijitcs.2012.2103.

14. Canadian Institute for Cybersecurity, https://www.unb.ca/cic/datasets/ids-2017.html 